\title{
Behaviour-based Relay Strategy on Mobile Ad-hoc Network for Effective Power Consumption
}

\author{
Hemalatha $\mathrm{S}^{1}$, Shobana $\mathrm{M}^{2}$, Sindhu $\mathrm{G}^{3}$, Anand $\mathrm{M}^{4}$, Anbarasan $\mathrm{M}^{5}$ \\ \{ pithemalatha@gmail.com ${ }^{1}$, shobanam@srmist.edu.in ${ }^{2}$, Sindhudhara@gmail.com³ \\ anandmenscall@gmail.com ${ }^{4}$, anbarasan.cse@gmail.com ${ }^{5}$ \}
}

Professor, Department of Computer Science and Engineering, Panimalar Institute of Technology, Chennai. ${ }^{1}$, Assistant Professor, Department of Computer Science and Engineering, SRM Institute of Science and Technology, Kattankulathur ${ }^{2}$, Assistant Professor, Department of Computer Science and Engineering, Saveetha School of Engineering, Saveetha Institute of Medical And Technical Sciences, Chennai $^{3}$, Assistant Professor, Department of Computer Science and Engineering, SRM Institute of Science and Technology, Kattankulathur ${ }^{4}$, Associate Professor, Department of Computer Science and Engineering, Sri Sairam Institute of Technology, Chennai $^{5}$

\begin{abstract}
Flooding is the most basic form of broadcast, in which an approaching signal is retransmitted once by each organization's base. The transmission storm problem is caused by the basic flood system in remote Ad-hoc networks.. Even so, in terms of asset usage, such as transmission capacity and electricity, this approach is inefficient. Another cross-breed scheme is presented in this paper, which incorporates different methods to minimize overhead and track energy consumption. We present a Behaviour-based Relay Strategy that makes use of adjacent radios' 1-bounce results. The use of a purpose of ensuring or position estimation to pinpoint the location of hubs is not needed in this plan. The forthcoming convention, unlike earlier researches, splits the company into separate categories based on their communication-energy ratios. As a result, a Gateway hub is one that receives HELLO messages from different gatherings. This hub is actively interested in adding RREQ parcels, avoiding the need for redundant data transfer. When compared to AODV, the proposed convention's exhibition evaluation indicates a reduction in steering overhead and energy use..
\end{abstract}

Keywords: AODV, Mobile Ad-hoc Network, Quality of Service, forwarding scheme.

\section{Introduction}

Versatile impromptu organization is fundamentally a gathering of portable hubs that impart utilizing multi-bounces in a non-framework based arrangement. Because of their enormous potential across both combat and general uses, mobile ad hoc networks have been receiving serious consideration in recent years [1][2][3]. In sending the control packets, the transitional hubs between origin and goal play an important role. This flooding component is basic to the course disclosure measure. Notwithstanding, flooding can be excess and energy burning-through. In this way, various attempts were undertaken to restrict the transmissions in controlling parcels in order to reduce the amount of capturing that was unnecessary.

Flooding is the very often utilized strategy for hubs to trade network data or convey Routing Request messages to an objective. Also, the most straightforward approach to flood an organization is through a strategy called Simple Flooding. When a hub receives the parcel, 
this can unintentionally start broadcasting it to its other one-jump neighbors, resulting in a large number of bundles being rebroadcast. As a result, several bundle crashes occur, particularly if the organization is large. As a result, the primary motivation behind various flooding methods in MANETs is to avoid the significant Transmitted Drainable even more than possible to retain high organization inclusion and low conveyance inertness.

The random Transmit Power values of the adjoining hubs are taken into account in this paper's power proficient behavior-based relay strategy. In view of the hub location, the proposed conspire gradually adjusts the RREQ probability of sending at each hub. However, in a sparsely populated area, this cornerstone is ignored in comparison to a heavily populated district in order to preserve organization availability. Following the receipt of a packet, the decision to advance RREQ bundles is taken in a flash. In this study, hilter kilter radio reaches were taken into account. The new strategy reduces unnecessary excess retransmission while also increasing the reachability of information parcels while also lowering the proportion of dropped bundles and package misfortune. As a result, the overall steering overhead is reduced, and energy utilization is improved. The standard AODV is compared to our work in order to evaluate our strategy.

\section{Related Work}

Because it's sort of distributed plot used throughout the organization's presentation is so significant, it must be carefully selected. There are several telecom conventions used in MANETs to achieve effective flooding and prevent excessive repeated retransmission.

All communication transmission across a remote medium in remote organizations is done through natural communication, which is not the same as the Web. Because of the nature of transmission, many opportunities based on catching can be used to increase the information transmission capacity in remote organizations. Ex-OR is the primary practical information sending plan that aims to improve information transmitting capacity by using transmission nature in remote lattice organizations, and pioneering information sending becomes a wellknown term used by Ex-OR to describe this type of modern information sending plan. ExOR's central idea has sparked many decisions. However, almost all of these deductions are used for remote cross-section organizations or involve the positioning administration to support crafty data sending in Mobile Ad-hoc Networks. In [4], the developers devised a series of solutions for executing sharp information sending in larger MANETs, dubbed Supportive Opportunistic Routing Scheme in Mobile Ad-hoc Networks through Restricted Scope Retransmission, which allows them to use transmission natures other than ExOR and, in addition, aids them in improving the proficiency and power of shrewd information sending in MANETs.

Instead of program and portfolio directly to the passage/door, the IoT system would be significantly more energy efficient if hubs forward bundles through closest neighbors in multibounce ways in the future. To spur the middle of the road hubs to transfer parcels, to compensate for the energy loss in hubs due to package transferring, Avirup Das et al suggested a fresh motivator system [5] for sharing broad band psychological range. The yield demonstrated that the developed plot requires electricity fundamentally and also maintains energy expenditure independent of such hand-off workload with such a negligible increase on testing workload when comparing direct organizations to its speculation for irregular organizations. 
In request to improve security, creators proposed the assessment of trustworthiness of hubs is critical. This work attempts to limit overhead through limiting repetitive information conveyance, expectation of connection disappointment and energy-mindful hub determination via looking On-Demand Multicast Routing Protocol plan and the conceivable fluffy improvement. In work [6], aggressor discovery is tried utilizing multi-specialist arrangement to refresh hub's trustworthiness for dynamic.

In article [7] writers guaranteed quality mindful directing and information sending in MANET correspondence, by giving ILR-GTCDW method. Two handling measures are performed by the ILR-GTCDW. Initially, steering was accomplished via entwined device guiding. To reduce steering workload and consider an accurate area of objective in ILR, assumption expansion and probabilistic strategy are used. Furthermore, hereditary geography control information sending is performed through agreeable correspondence. The ideal steering way is picked by utilizing the hereditary calculation based Topology control Data sending procedure that gives both solid information sending and negligible energy utilization.

All together for multicast information parcels to be communicated productively, the multicast information sending is needed at every switch. Inside a wired organization, an organization interface of a hub has a coordinated association with one of the approaching interfaces of different hubs. If a package arriving at an approaching gateway needs to be sent, this is sent to the contrasting make recommendations. Nonetheless, in a portable impromptu organization, by and large every hub has just one organization interface, so the approaching interface is equivalent to the active one and the remote organization interface of a hub has a one-to-numerous association with those of adjoining hubs. This distinction may cause issues, for example, directing circles and parcel duplication. In this paper [8], Youngmin Kim et al proposed a multicast information sending plan which can be utilized in the multi-bounce remote specially appointed organization without causing either directing circles or parcel duplication. A list is specified in this plan to prevent parcel redundancy, which can occur when another leaf multihop steering convention would be used.

\section{Behaviour-Based Relay Strategy}

The forward hub decision is critical to the proposed calculation's preparation. As a result, we classify the organization's diverse hubs as entry point or Non-entry point in terms of the adjacent Transmission-Power level in this study (PtLevel). Entry point, which have numerous PtLevel upsides in their adjacent table, are obligated to advance RREQ packages. Non- entry point, on the other hand, are hubs that have the same PtLevel advantages in their adjacent table. Every transmission by Non-entry point must be excluded to controlled delivery excessive repetitive data transfer and to reduce steering parcel payload. As a result, the hub location determines whether the hub is an Entry point or a Non-entry point. Portals are hubs that are situated between different gatherings (and receive bundles from various PtLevel upsides); otherwise, they are known as Non-entry point.

The procedure of calculating their various PtLevel advantages is used [3], which involves progressively changing the Communication Cost on every hub that brings the majority of neighbors inside a predetermined force level. The hub's Transmit Power value varies in the actual sheet. The basic idea is that only certain hubs are required to advance the package, which extends to groups of hubs with varying PtLevel. Intuitively, a center with a similar 
PtLevel to its neighbors is almost certainly located within the same gathering. As a result, all of your neighbors can receive a transmission package.

However, in order to maintain organization availability, Both for hub that is located in a high-inadequate or opens up the potential area, that foundation for entryway assurance is overlooked (every one of its neighbors has the same PtLevel and there is no hub designated as passage). The PtLevel is then used in the proposed technique to achieve more proficient and heavy flooding.

At the point when the hubs information is utilized to decide whether it ought to take part in RREQ sending, two objectives are accomplished. Above all else, energy that is the fundamental asset of hubs will be saved, in light of the fact that solitary explicit hubs can take an interest in re-sending RREQ parcels. Furthermore, the effect of expanding control overheads and clog in remote organization, which could prompt debasing of the organizations execution, are stayed away from. This section will go over the proposed Relay Strategy in detail.

\section{a. Control Packets}

We need to exchange parcels to determine whether the organization's hub is a Portal or otherwise, and instead of inserting new controlling bundles, we alter the existing control bundles in the AODV convention [9] for convey their PtLevel and Status.

HELLO parcel: To exchange data about their transmission-power level and status, all hubs as in suggested convention use the HELLO package of AODV. For collect this information, the HELLO parcel has two new fields: communication-energy scale (PtLevel) or Rank. Each hub creates a neighbor table also with help of the HELLO package, which stores the PtLevel or Rank advantages of each 1-bounce neighbor.

\section{b. Route Discovery}

All hubs, including the initial hub, moderate hubs, and objective hub, are subject to the course disclosure measure.

The proposed conspiracy's disclosure period is as shown in:

Initial Hub: The source cycle is like except AODV, in that it occurs if an origin requires a path towards the target. If a origin hub needs to transfer packages to an objective hub but there is no available route, the origin hub can send an RREQ parcel that find another way to the objective.

Transitional Hub: In any halfway hub inside the origin communication region gets the RREQ parcel interestingly, a hub carrying out the proposed system that appeared in Fig. 1. 


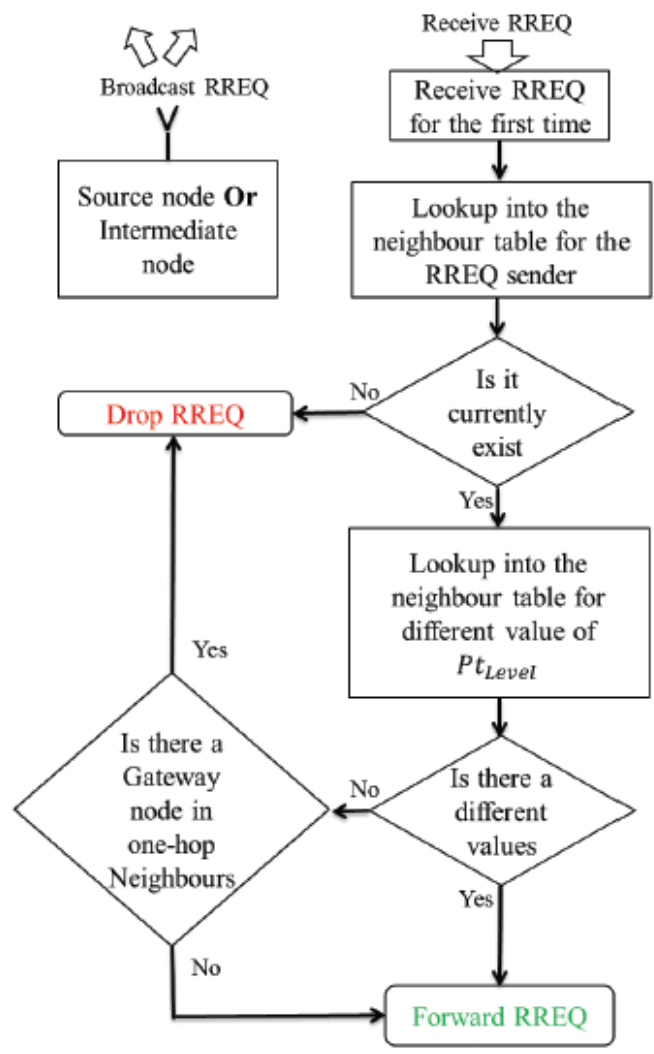

Fig.1 Relay Strategy for RREQ

Objective Node: When the RREQ package arrives at the expected objective or a transitional hub with a new path towards the objective, these single routes a response by transmitting a Route Reply (RREP) parcel all along opposing way developed at moderate hubs in during course disclosure measure. Nonetheless, we noticed an excessive number of information parcels dropped by the directing layer while carrying out the proposed calculation on the Fig.2. 


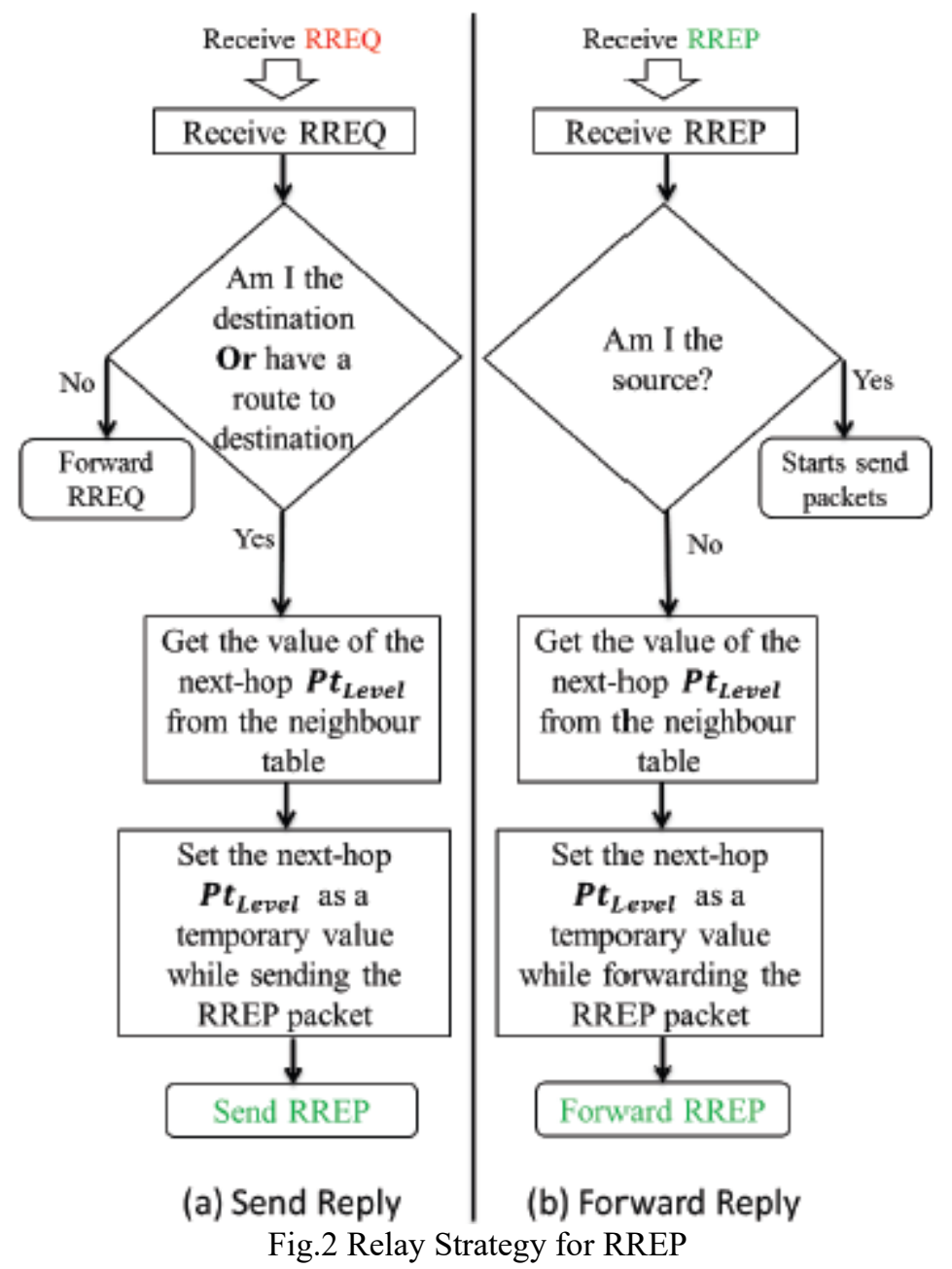

\section{Evaluation Metrics And Simulation Parameters}

The NS2.35 framework is used for reenactment as well as execution evaluation. The specific reenactment boundaries can be found in Table I. The portability of the hub was achieved by using an arbitrary waypoint models for different speeds and indeed the portability seemed to have no significant effect on execution. The reproduction used the UDP (User Datagram Protocol) including Constant Bit Rate association model (CBR). The assessment is based on the reenactment with various numbers of hubs $(20,40,60,80$ and 100) that structure an organization across a given bandwidth of $1000 \mathrm{~m} \times 1000 \mathrm{~m}$. The duration of the replication is set to 100 seconds. Furthermore, the IEEE 802.11 layer was used. This boundary was commonly used in previous studies, allowing the outcomes of this reenactment to be compared to the findings of those studies. 
Table I. Simulation Parameters

\begin{tabular}{c|c}
\hline Mac Layer & IEEE 802.11 \\
\hline Bandwidth & $2 \mathrm{Mb} / \mathrm{s}$ \\
\hline Propagation model & Two-ray ground \\
\hline Antenna model & Omni-Antenna \\
\hline Simulation area & $1000 \mathrm{~m} \times 1000 \mathrm{~m}$ \\
\hline Simulation time & $100(\mathrm{sec})$ \\
\hline Number of nodes & $75,100,150,200$ \\
\hline Node speed & $10,20,30(\mathrm{~m} / \mathrm{s})$ \\
\hline Pause time & $40(\mathrm{sec})$ \\
\hline Traffic type & $\mathrm{CBR}$ \\
\hline Number of connections & 40
\end{tabular}

To dissect the exhibition of our plan, we contrast our Behavior-based Relay Strategy and AODV, which outflanks AODV in the entirety of our reenactment results shown in Fig. 3,4,5.

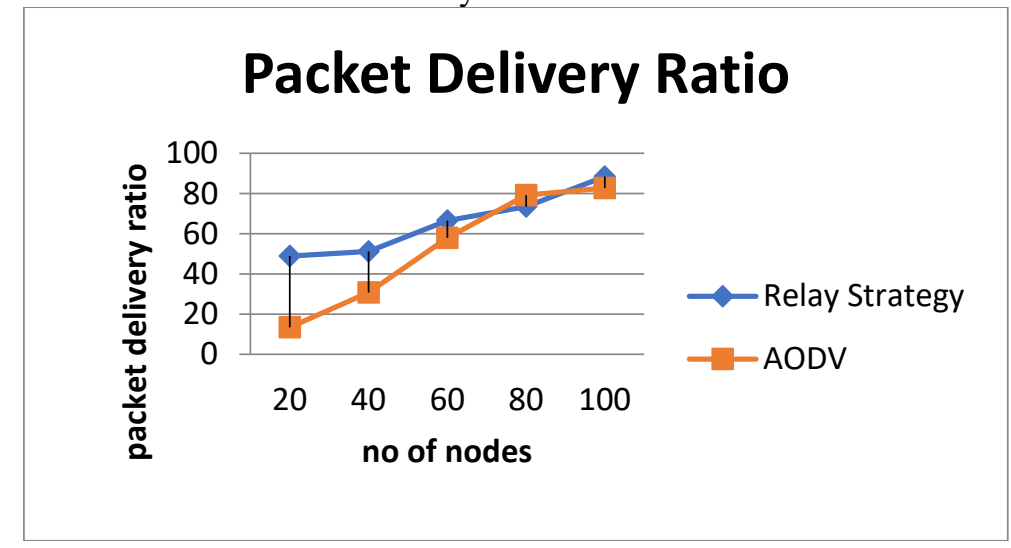

Fig.3 Packet Delivery Ratio

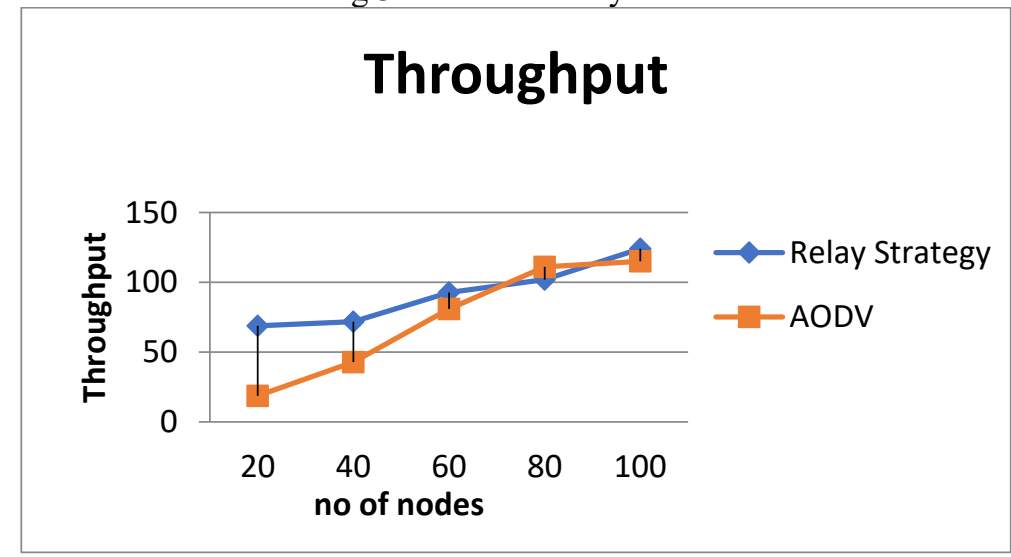

Fig. 4 Throughput 


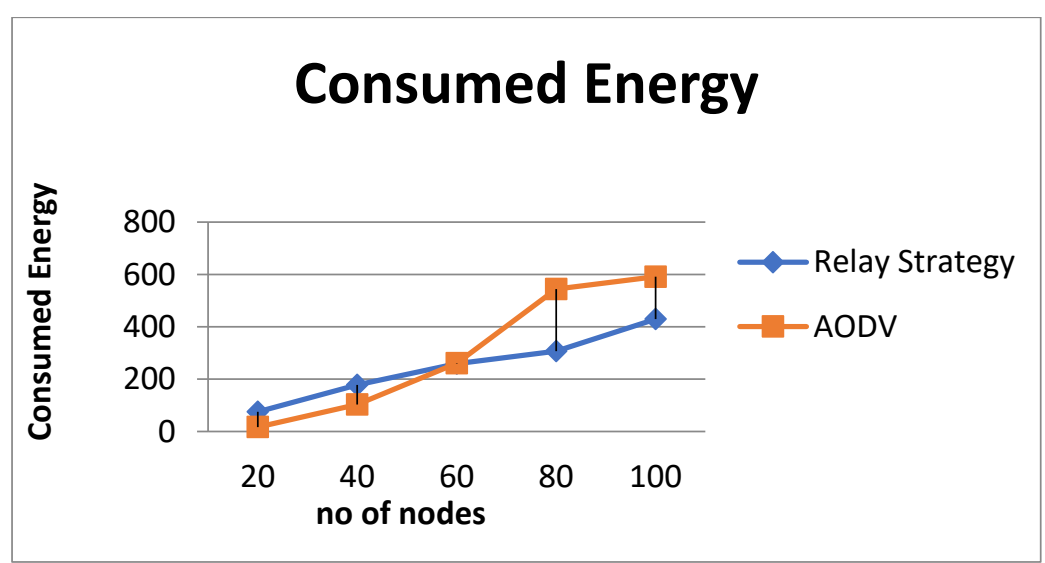

Fig.5 Consumed Energy

\section{Conclusion And Future Work}

Despite the fact that energy consumption are being reduced just at device scale, at the communication scale, and using an energy guiding convention, this paper proposed a novel crossover scheme that combines multiple procedures that work together to reduce workload and moderate power consumption. The procedures utilized are: 1) Using communicationenergy monitoring system to diminish impedance among hubs and increment the throughput in the organization, hence decreasing the general energy utilization. 2) Developing Transmission-Power mindful directing calculation to confine rebroadcast RREQ bundles to keep away from superfluous catching, and choosing the proper force level to convey the RREP parcels to its objective; 3) Avoiding cycles that rely heavily on the Geolocation and distances adding devices that squanders power and calculation assets at gadget scale.

Further enhancement and upgrade for reducing clog and power use will be planned in our future research. For example, when door hub set is resolved, the issue of how to plan those entryway hubs to proficiently advance RREQ parcels requires further investigation.

\section{References}

[1] S. Banerjee, A. Ghosh, S. Mahapatra and R. Karmakar, "Performance Survey of MANET Routing Protocols with TCP Congestion Control Algorithms," 2020 IEEE 1st International Conference for Convergence in Engineering (ICCE), Kolkata, India, 2020, pp. 433-438, doi: 10.1109/ICCE50343.2020.9290610.

[2] Anand, M., Sasikala, T. Efficient energy optimization in mobile ad hoc network (MANET) using better-quality AODV protocol. Cluster Comput 22, 12681-12687 (2019). https://doi.org/10.1007/s10586-018-1721-2

[3] M. Anand, N. Balaji, N. Bharathiraja et al., A controlled framework for reliable multicast routing protocol in mobile ad hoc network, Materials Today: Proceedings, https://doi.org/10.1016/j.matpr.2020.10.902

[4] S. S. Kumar and V. Parthasarathy, "A new supportive opportunistic routing scheme in Mobile Ad-hoc Networks," 2013 International Conference on Current Trends in Engineering and Technology (ICCTET), 2013, pp. 174-176, doi: 10.1109/ICCTET.2013.6675938. 
[5] A. Das, N. Das, A. Das Barman and S. Dhar, "Energy Incentive for Packet Relay Using Cognitive Radio in IoT Networks," in IEEE Communications Letters, vol. 23, no. 9, pp. 15811585, Sept. 2019, doi: 10.1109/LCOMM.2019.2923233.

[6] Gayathri Devi S., Marimuthu A. (2019) Honest Forwarding Node Selection with Less Overhead and Stable Path Formation in MANET. In: Peter J., Alavi A., Javadi B. (eds) Advances in Big Data and Cloud Computing. Advances in Intelligent Systems and Computing, vol 750. Springer, Singapore. https://doi.org/10.1007/978-981-13-1882-5_41

[7] Murugan, K., Anita, R. Interlaced Link Routing and Genetic Topology Control Data Forwarding for Quality Aware MANET Communication. Wireless Pers Commun 102, 3323 3341 (2018). https://doi.org/10.1007/s11277-018-5370-9

[8] Kim Y., Ahn S., Lee J. (2005) An Efficient Multicast Data Forwarding Scheme for Mobile Ad Hoc Networks. In: Kim C. (eds) Information Networking. Convergence in Broadband and Mobile Networking. ICOIN 2005. Lecture Notes in Computer Science, vol 3391. Springer, Berlin, Heidelberg. https://doi.org/10.1007/978-3-540-30582-8_53

[9] Anand M, Sasikala T, Anbarasan M. Energy efficient channel aware multipath routing protocol formobile ad-hoc network. Concurrency Computat Pract Exper. 2019;31:e4940. https://doi.org/10.1002/cpe.4940 